Conclusions High LDL/oxLDL levels by Apoe deficiency affect bone destruction during AIA by reducing the number of osteoclasts in the synovium, probably by interfering with osteoclastogenesis.

Disclosure of interest None declared

\section{P051 LOSS OF BONE MASS IN PATIENTS WITH HEAD AND NECK TUMOURS IN THE FIRST YEAR AFTER THE DIAGNOSIS OF THE DISEASE}

${ }^{1} \mathrm{R}$ Hernández*, ${ }^{2} \mathrm{~J}$ Fuentes Pradera, ${ }^{1} \mathrm{~J}$ Uceda, ${ }^{1} \mathrm{JL}$ Marenco de la Fuente. ${ }^{1}$ Rheumatology; ${ }^{2}$ Oncology, Valme University Hospital, Seville, Spain

\subsection{6/annrheumdis-2018-EWRR2018.70}

Introduction Head and neck tumours (TTCC) constitute a heterogeneous group of diseases that affect the otorhinolaryngological sphere. The risk factors such as tobacco and alcohol are also for osteoporosis too. The cancer itself, with the malnutrition suffered after surgical treatment and chemoradiotherapy, represents a potential situation of bone loss with the subsequent appearance of osteoporosis and its complication 'the fracture'. We do not have data on the frequency of osteoporosis in patients with head and neck tumours.

Objectives To evaluate the loss of bone mineral density (BMD) in the lumbar spine during the first year of follow-up after diagnosis in a cohort of patients with head and neck tumours. Methods Observational, longitudinal and prospective study in a cohort of patients with TTCC at the Virgen de Valme hospital, one year follow-up. Bone mineral density was measured by DXA in the lumbar spine before the start of treatment and once a year. To identify qualitative factors that determine bone loss $(<2 \% / \geq 2 \%)$, contingency tables are performed and the Chi-Square test or the non-asymptotic methods of the Monte Carlo test and the Exacta test are applied.

Results A total of 50 patients were included. $100 \%$ of the patients were male. The average age at diagnosis of $60.9 \pm 7.8$ years. $98 \%$ were smokers, and $76 \%$ habitual heavy drinkers. $12 \%$ had a family history of hip fracture. $18 \%$ have a disease that causes malabsorption syndrome.

The median BMD at baseline is $1.05 \mathrm{~g} / \mathrm{cm}^{2}$, and after one year of follow-up this median decrease is 1.02. The loss of bone mass in a year expressed in median is estimated at 0.03 $\mathrm{g} / \mathrm{cm}^{2}$, which corresponds to a loss of bone mass per year of $2.8 \%$, which reflects a greater loss than that of a population of same age and sex estimated at $0.5-1 \%$ per year.

Although the loss suffered by our patients is not from a significant statistical point of view, given the sample size, it does reflect a tendency to increase the risk of osteoporosis in these patients.

Conclusions Patients with TTCC suffer a bone loss greater than expected in a population of equal age and sex, in the first year of follow-up. Initial screening of these patients would be indicated, which would include OP and/or fracture risk factors.

\section{REFERENCE}

1. Valero C, Olmos JM, Rivera F, Hernandez JL, Vega ME, Gonzalez Macias J. Osteoprotegerin and bone mass in squamous cell head and neck cancer patients. Calcif Tissue Int 2006;78:343-347.

Disclosure of interest None declared

\section{P052 EFFECTS OF HYPOXIA AND ACTIVATED HUMAN B CELLS ON IMMUNOSUPPRESSIVE AND DIFFERENTIATION CAPACITY OF MULTIPOTENT MESENCHYMAL STROMAL CELLS}

${ }^{1} \mathrm{R}$ Scarpone*, ${ }^{1} \mathrm{P}$ Gitsioudis, ${ }^{2} \mathrm{R}$ Saffrich, 'L Tykocinski, ${ }^{1} \mathrm{H}-\mathrm{M}$ Lorenz, ${ }^{1} \mathrm{~T}$ Tretter. ${ }^{1}$ Dept. Medicine V, Div. Rheumatology; ${ }^{2}$ Dept. Medicine V, Div. Haematology, University Heidelberg, Heidelberg, Germany

\subsection{6/annrheumdis-2018-EWRR2018.71}

Introduction Multipotent mesenchymal stromal cells (MSCs) are a promising approach for the treatment of chronic inflammatory autoimmune diseases. However, little is known about whether their immunomodulatory, replicative and regenerative potential is influenced by the pathogenic environment.

Objectives Activated lymphocytes and hypoxia are part of the cellular environment in chronic inflammatory diseases. The aim of the study was to investigate the influence of activated $\mathrm{B}$ cells and hypoxia on MSCs functions.

Methods Bone marrow MSCs from healthy donors were cultured for various time periods under normoxia or hypoxia $\left(\begin{array}{ll}3 \% & \mathrm{O}_{2}\end{array}\right)$ in presence or absence of $\mathrm{B}$ cells from healthy donors activated via B cell receptor cross-linking and IL-2. Alternatively, cell culture supernatant from B cells activated for three days with respective stimuli was used. Cell division and expression of surface markers were assessed by flow cytometry, cytokine secretion by ELISA and gene expression by qRT-PCR. For differentiation, MSCs were cultured for 21 days in established differentiation media in presence or absence of B cell culture supernatant. Osteogenesis and adipogenesis were assessed by fluorescence microscopy and quantified with ImageJ software.

Results Significant increase of MSC IL-6 and IL-8 production over more than 100-fold in coculture with B cells revealed activation of MSCs by $B$ cells both under normoxic and hypoxic conditions. In contrast, B cell specific TNF- $\alpha$ production was reduced by more than half, demonstrating suppression of B cell activation by activated MSCs in return. This was observed both in cocultures with direct contact and indirect contact upon separation by cell culture inserts, suggesting soluble factors were sufficient for observed effects. Both hypoxic conditions and coculture with MSCs significantly inhibited B cell division, without increasing apoptosis. B cell CD25 expression significantly decreased in cocultures, but remained higher under hypoxia. B cell supernatant increased MSC proliferation rate and delayed entry into senescence and this effect was more pronounced under hypoxia. Of note, B cell-derived factors increased MSC-specific expression of hypoxia induced transcription factor HIF- $1 \alpha$ under normoxic conditions. B cell derived factors also supported osteogenic differentiation and inhibited adipogenesis both under normoxia and hypoxia.

Conclusions These results suggest an important influence of the inflammatory environment on MSCs functions: In presence of B cells MSCs exert immunosuppressive properties, but also secrete high amounts of proinflammatory cytokines. In addition, the replicative potential of MSCs increases, which is further supported by hypoxia. In presence of activated B cells, MSCs differentiation is governed towards the osteogenic lineage and this effect is stable both under normoxia and hypoxia.

Disclosure of interest None declared 